\title{
How to eat when I can't swallow?
}

\section{Dominika Lisiecka}

\author{
Department of Speech \& Hearing Sciences, University College Cork, UCC
}

\begin{abstract}
"I had another choking session last evening. A tiny bit of salmon or salad went down the wrong way and I coughed and coughed with my loved one beating my back with a vengeance. This went on for the best part of two hours." (Mark Cato, diagnosed with Motor Neuron Disease).
\end{abstract}

\section{Background}

Imagine losing control over the muscles of your mouth and throat. Imagine not being able to chew or swallow. Imagine life without a burger or a chocolate cake. Imagine fits of coughing when you try to swallow anything. Imagine looking at food with fear, fear of choking.

Swallow difficulties can affect anyone and at any stage of life. It can be a congenital disorder - for example caused by cerebral palsy, or it can be acquired - caused by a stroke, cancer, or progressive neurological illness. When the mouth muscles stop working properly you may experience difficulties chewing food. Eating and drinking may become a very long, effortful and messy process. It can make you feel embarrassed, especially when you eat in public. When the throat muscles are affected you are at risk of choking and aspiration. This means that any drink, food or even saliva may redirect towards your lungs instead of flowing smoothly into the stomach. Long term, it can cause chest infection, aspiration pneumonia or death.

When it is not longer possible to eat and drink safely some people decide to have a feeding tube inserted and take food directly into the stomach. Others continue eating despite serious risks. The decision is always individual - an universal set of guidelines stating the appropriateness of non-oral feeding for every medical condition does not exist. Everyone has unique circumstances, which have to be considered carefully. Patient's prognosis may be an important factor: it may be "easier" to accept artificial feeding temporarily, while waiting for the swallow to improve. However, the perspective can be very different for people diagnosed with a progressive illness, for which there is no cure, such as Motor Neuron Disease (MND).

MND is a rare neurodegenerative illness of unknown etiology. It affects more than swallowing. It can cause weakness of any muscle in the body. The disease is extremely rapid. Median survival of Irish patients is just over 16 months after diagnosis. It is now recognised that MND is a multisystem disorder, which can lead to muscular weakness as well as 
cognitive impairment. Swallowing problems (dysphagia) can often occur in MND. They can have various medical consequences, like malnutrition, dehydration, chest infections, aspiration pneumonia, choking and death.

We know that appropriate nutrition is an important prognostic factor in MND. Therefore it is important to ensure adequate food intake, which may not be easy if a person has difficulties with swallowing.

Dysphagia can affect people not only from a medical, but also from psychological and emotional perspective. It is a complex problem, which is often managed in busy clinics with little time available for in-depth discussions. We have to understand how people with MND experience difficulties with eating and drinking, how they cope and how they feel. Dysphagia in MND is very specific due to the nature of the disease and can progress fast and can often occur in addition to other concomitant impairments/ disabilities. It is crucial to investigate this problem in-depth in order to know how to provide effective support for both people diagnosed with MND and their caregivers.

People living with MND require extensive support which creates substantial challenges for those caring for them. Caring duties can include preparation of meals and feeding. The caregivers are often expected to know how to alter food consistency to make it easier to swallow, how to feed safely, and how to react in case of a choking episode. How hard it can be to feed someone with impaired swallow, when on the one hand you know that nutrition is very important, but on the other you are afraid of them choking? How hard it is to watch someone, who can not have their usual diet anymore?

(...) I mean for instance, my daughter and I had a curry. And it sounds stupid, doesn't it? I had a curry, and very enjoyable, obviously. Suddenly my wife, in the kitchen, suddenly broke down in tears, and I had to go out and find out what was wrong. And after quite a long time it took to find out that she just said, "I wish I could partake of the curry." So that makes me feel pretty awful to think that I can enjoy a curry and my daughter can enjoy a curry, which is just normal, and my wife is not able to. So I suggested to her that she should try some of the liquids from the curry and everything. She tried, but then she informed me that it was a bit too hot, and the Madras curry or something was too hot and it was burning her throat and that. So again we tried to get round the problem a little bit, but it doesn't work. So, you know, what can you do? How do you approach it? (a Caregiver of a person with MND).

The experiences of the person living with MND and dysphagia have not been investigated. My research aims to address the above gap. I decided to focus on MND, as this disease progresses so fast and does not allow for any time to be wasted. Having deeper understanding of the problem means that it can be approached in a more efficient and adequate way.

My research proposal also aligns with the top 20 research priorities identified for Speech \& Language Therapists in Ireland by the Health Research Board. 


\section{Research aim}

The overall aim of this research is to gain a deeper understanding of how people living with MND experience dysphagia. This project seeks to investigate:

1. The impact of dysphagia on the lives of people with MND and their caregivers.

2. The main dysphagia related difficulties experienced by people with MND and their caregivers.

3. The meaning of dysphagia for people with MND and their caregivers (when it is often one of many disabilities).

4. How people with MND and their caregivers perceive dysphagia related services available to them, and their suggestions of any potential changes to these services.

\section{Data collection}

This is a qualitative research study, using a phenomenological approach for data collection and analysis. Phenomenology (study of experiences) allows in-depth investigation of what it is like to live with/ care for MND and dysphagia. A semi-structured interview design was chosen to provide in depth rich information pertaining to participants' experiences and help the researcher understand the participants in their own environment. The goal is to have the participant reconstruct their own experience within the topic under study.

A pilot test will be carried out to facilitate evaluation of the interview design and to allow for revision prior to implementation of the study, if necessary. One individual with MND and dysphagia and one caregiver will be enrolled in the pilot, following which a review of research design will be undertaken, as appropriate.

\section{Sample size}

It is expected to enrol 20 participants in total: 10 adults with MND and 10 caregivers.

\section{Data collection}

Data will be collected via multiple interviews. It is anticipated that about 60 interviews will be carried out. At least two (maximum 4) interviews are planned with each participant: to establish rapport and obtain in-depth information. Multiple interviews may also be required if participants become fatigued. 


\section{Data analysis}

The interviews will be audio recorded and transcribed verbatim. During the first phase of analysis the text will be read multiple times to develop emerging themes and to search for connections between them. This process will be repeated individually for each participant. The second phase will involve looking for patterns (common themes) across the group of adults with MND and the caregivers. The findings will be described and interpreted, as consistent with Interpretative Phenomenological Analysis. Credibility will be sought by design checking, member validation and triangulation.

\section{Recruitment}

The participants will be recruited from the Irish MND Association (IMNDA) and Speech \& Language Therapy Departments in Kerry /Munster and assessed accordingly to specific inclusion and exclusion criteria. The Kerry area was chosen as a convenient geographical location to ensure that multiple in depth interviews can be possible within the limits of financial and time resources. However, the research may expand to other parts of Munster if required.

\section{The importance of this project}

This innovative research project is of direct relevance to all people with MND, their caregivers, families and multiple clinicians involved in MND care, for example, Speech \& Language Therapists, Occupational Therapists, Dietitians, Palliative Health Nurses and Doctors. Little is known about Irish people living with MND, therefore this research will be of particular relevance to the Irish health care system and ultimately Irish Society. It will provide insight into the experiences of people with dysphagia in MND, which may explain some decisions made by patients (for example when refusing non-oral feeding). This study can result in improved quality of life and quality of care for MND patients in Ireland, for example, with appropriate level of support MND patients could potentially be better cared for in their own homes. It could also potentially identify where services are providing excellent care (meeting the needs of this population) as well as where there are gaps. Without qualitative studies such as this, a "real" understanding of MND can not be achieved.

The unexamined life is not worth living (Socrates). 